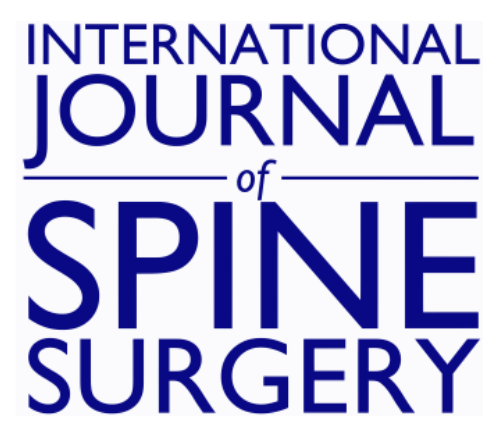

\title{
Assessing the Quality and Credibility of Publicly Available Videos on Cervical Fusion: Is YouTube a Reliable Educational Tool?
}

James D. Baker, Younus Baig, Zakariah K. Siyaji, Alexander L. Hornung, Athan G. Zavras, G. Michael Mallow, Shadi Zbeidi, Nicholas A. Shepard and Arash J. Sayari

Int J Spine Surg 2021, 15 (4) 669-675

doi: https://doi.org/10.14444/8088

http://ijssurgery.com/content/15/4/669

This information is current as of April 26, 2023.

Email Alerts Receive free email-alerts when new articles cite this article. Sign up at: http://ijssurgery.com/alerts 


\title{
Assessing the Quality and Credibility of Publicly Available Videos on Cervical Fusion: Is YouTube a Reliable Educational Tool?
}

JAMES D. BAKER, BA, YOUNUS BAIG, MA, ZAKARIAH K. SIYAJI, BS, ALEXANDER L. HORNUNG, BS, ATHAN G. ZAVRAS, BS, G. MICHAEL MALLOW, BS, SHADI ZBEIDI, BS, NICHOLAS A. SHEPARD, MD, ARASH J. SAYARI, MD

Department of Orthopaedic Surgery, Division of Spine Surgery, Rush University Medical Center, Chicago, Illinois

\begin{abstract}
Background: YouTube has become a popular source for patient education, though there are concerns regarding the quality and reliability of videos related to orthopaedic and neurosurgical procedures. This study aims to evaluate the credibility and educational content of videos on YouTube related to cervical fusion. Secondarily, the study aims to identify factors predictive of higher or lower quality videos.

Methods: A YouTube query using the search terms "cervical fusion" was performed, and the first 50 videos were included for analysis. Reliability was assessed using the Journal of the American Medical Association (JAMA) criteria. Educational quality was assessed using the Global Quality Score (GQS) and the Cervical Fusion Content Score (CFCS). Videos were stratified by content and source, and differences in JAMA, GQS, and CFCS scores were assessed. Multivariable linear regression was used to identify predictors of higher or lower JAMA, GQS, and CFCS scores. Statistical significance was established at $P<0.05$.

Results: Total number of views was 6221816 with a mean of $124436.32 \pm 412883.32$ views per video. Physicians, academic, and medical sources had significantly higher mean $J A M A$ scores $(P=0.042)$. Exercise training and nonsurgical management videos had significantly higher mean CFCS scores $(P=0.018)$. Videos by physicians $(\beta=$ 0.616; $P=0.025)$ were independently associated with higher $J A M A$ scores. Advertisements were significant predictors of worse CFCS $(\beta=-3.978 ; P=0.030)$, and videos by commercial sources predicted significantly lower $J A M A$ scores $(\beta=$ $-1.326 ; P=0.006)$.

Conclusions: While videos related to cervical fusion amassed a large viewership, they were poor in both quality and reliability. Videos by physicians were associated with higher reliability scores relative to other sources, whereas commercial sources and advertisements had significantly lower reliability and educational content scores. Currently, YouTube seems to be an unreliable source of information on cervical fusion for patients.

Level of Evidence: 4.

Clinical Relevance: The results of this study aid surgeons in counseling patients interested in cervical fusion, and suggest that publicly available videos regarding cervical fusion may not be an adequate tool for patient education at this time.
\end{abstract}

Cervical Spine

Keywords: cervical, fusion, patient education, video, surgery

\section{INTRODUCTION}

While physicians and health care providers have traditionally been the primary source of medical information, an increasing number of patients are turning to the Internet as a source for health care guidance. It has previously been reported that $61 \%$ of adults in the United States regularly search for health-related content, and approximately $80 \%$ of adults have turned to it at least once. ${ }^{1,2}$ YouTube, an online open-source platform that allows users to view and upload videos, is widely regarded as the most popular Web site where users can view videos and share information.

Videos related to health care issues are becoming more popular; however, there has been concern over the quality and reliability of these videos for patient education. ${ }^{3,4}$ Several studies have reported overall poor educational quality of videos pertaining to a variety of orthopaedic and neurosurgical conditions and procedures, including femoroacetabular impingement syndrome, ${ }^{3}$ knee arthroplasty, ${ }^{4}$ scolio- 
sis, ${ }^{5}$ posterior cruciate ligament injuries, ${ }^{6}$ and others. The risk of misinformation is of particular concern to spine surgeons, as $54 \%$ of patients report researching their condition before consultation with orthopaedic or neurosurgical surgeons. ${ }^{7}$

Cervical spondylosis is fairly common, with approximately $50 \%$ of patients over 40 years old and $85 \%$ of patients over 65 years old having some evidence of cervical degeneration. ${ }^{8,9}$ Though many patients remain asymptomatic, ${ }^{8,10}$ roughly 83 per 100000 individuals will develop cervical radiculopathy every year. ${ }^{11}$ Cervical fusion is a commonly used surgical procedure for degenerative spine pathologies, including radiculopathy, cervical myelopathy, and degenerative disc disease. Given the high prevalence of cervical degeneration, the annual number of cervical spinal surgeries has been increasing over time. ${ }^{12}$ As more patients are referred for surgery, it is imperative that accurate and valuable information be available for patients who are contemplating surgery.

Therefore, the current study aims to assess the educational quality and reliability of publicly available YouTube videos related to cervical fusion. Secondarily, this study aims to identify factors associated with higher and lower quality videos.

\section{METHODS}

\section{YouTube Search}

An online query was performed on the YouTube online library (https://www.youtube.com/) using the keywords "cervical fusion." The first 50 video results were recorded and assessed for use in the study using a method previously accepted in other peer-reviewed literature. ${ }^{6,13}$ Exclusion criteria for videos included non-English videos or audio-only soundtracks. In cases where a video was excluded, the next consecutive eligible video was included for consideration.

\section{Video Characteristics}

For each video result, the following video characteristics were recorded to be analyzed: (1) title, (2) video source or uploader, (3) type of content, (4) video duration, (5) days since upload, (6) number of views, (7) view ratio (views/day), (8) number of likes, (9) number of dislikes, (10) like ratio (like*100/like + dislike), and (11) video power index (VPI; like ratio*view ratio/100). The VPI,
Table 1. The Journal of the American Medical Association score criteria and descriptions.

\begin{tabular}{ll}
\hline Criteria & \multicolumn{1}{c}{ Description } \\
\hline Authorship & $\begin{array}{c}\text { Author and contributor credentials and affiliations are } \\
\text { clearly stated } \\
\text { Clearly lists all copyright information and includes } \\
\text { references or sources for content } \\
\text { Date of post and subsequent updates to content are } \\
\text { included }\end{array}$ \\
Currency & $\begin{array}{c}\text { Conflicts of interest, funding, sponsorship, advertising, } \\
\text { support, and video ownership are disclosed }\end{array}$ \\
\hline
\end{tabular}

which has been used in previous literature, is a measurement that represents video popularity. ${ }^{6,13}$

\section{Video Upload Sources}

Video sources or uploaders were broken down into the following categories: (1) academic (authors or uploaders with research or university or college affiliations), (2) physician (independent physicians or physician groups without research or university or college affiliations), (3) nonphysicians (health professionals other than licensed medical doctors), (4) athletic trainers, (5) medical sources (content or animations from health-focused Web sites), (6) patients, and (7) commercial sources.

\section{Video Content Categories}

Video content was classified into the following categories: (1) exercise training (videos on rehabilitation and therapy post cervical fusion), (2) general information related to cervical fusion, (3) patient testimonials, (4) surgical technique, (5) nonsurgical management, and (6) advertisements.

\section{Assessment of Video Reliability and Educational Content Quality}

The Journal of the American Medical Association $(J A M A)$ benchmark criteria were used to assess the accuracy and reliability of the video results. The $J A M A$ benchmark criteria (Table 1) are a nonspecific and objective set of 4 guidelines that may be identifiable in online videos and resources. These criteria include (1) authorship, (2) attribution, (3) currency, and (4) disclosure. The observer assigns 1 point for each criterion fulfilled. Authorship criteria assesses the quality of the authors, contributors, academic affiliation, and credentials. Attribution assesses the references and sources used, as well as the copyright information. Currency evaluates the date content is posted and its use of up-to-date information. Finally, disclosure assesses any spon- 
Table 2. The Global Quality Score.

\begin{tabular}{ll}
\hline Grade & Description \\
\hline 1 & $\begin{array}{c}\text { Poor quality; not useful for patient education } \\
2\end{array}$ \\
$\begin{array}{c}\text { Poor quality; minimal relevant information. Limited utility to } \\
\text { patients }\end{array}$ \\
3 & $\begin{array}{c}\text { Suboptimal quality; some useful information present, but } \\
\text { missing key topics. Somewhat useful to patients }\end{array}$ \\
4 & $\begin{array}{c}\text { Good quality; most important topics discussed. Useful to } \\
\text { patients }\end{array}$ \\
5 & $\begin{array}{c}\text { Excellent quality; all topics covered in a clear manner. Highly } \\
\text { useful to patients }\end{array}$ \\
\hline
\end{tabular}

sorship, commercial funding, advertisements, or other potential conflicts of interest. A total score of 0 represents low accuracy and reliability, whereas a total score of 4 represents high accuracy and reliability. Though this method is not validated, it has been used previously in peer-reviewed literature as a means of assessing the reliability of online resources. ${ }^{6,13,14}$

To assess the overall educational content quality of the videos, we used the Global Quality Score (GQS). The GQS (Table 2) is a ranking tool ranging from poor quality (not educationally useful to patients) to excellent quality and flow (highly useful to patients). Scores range from 1 to 5 with a maximum score of 5 indicating high educational quality. Like the JAMA score, the GQS has not been validated, but it has been used in previous peer-reviewed literature to assess the content quality of online resources. ${ }^{6,13,14}$

To assess educational content quality specifically related to cervical fusion, we created the Cervical Fusion Content Score (CFCS). This 16-item tool is based on guidelines published by the American Academy of Orthopaedic Surgeons. ${ }^{15}$ Although this is a nonvalidated tool, similar methods for assessing the educational quality of online videos using orthopaedic and neurosurgical topic-based instruments have been noted in previous peer-reviewed literature. ${ }^{6,16}$ The CFCS criteria (Table 3) include information pertaining to (1) common patient symptoms and populations; (2) general information about cervical fusion; (3) diagnoses and evaluations warranting cervical fusion; (4) methods, risks, and benefits pertaining to cervical fusion surgeries; and (5) postoperative outcomes. The observer assigns 1 point for each criterion satisfied with a maximum possible score of 16 indicating high cervical fusionspecific educational content quality. Interrater reliability for all 3 outcome tools was assessed using intraclass correlation (ICC) analysis. For the
Table 3. Criteria assessed in the cervical fusion content score.

Patient presentation

Describes symptoms

Describes relevant patient population

General information

Defines cervical fusion

Compares cervical fusion to lumbar fusion

Explains purpose of cervical fusion

Mentions majority population affected

Diagnosis and evaluation

Mentions specific or relevant symptoms

Discusses use of imaging

Mentions red flag requiring urgent treatment

Treatment

Describes multiple surgical approaches

Describes the use of bone grafting

Describes the associated risks

Describes the associated benefits

Outcomes

Discusses postoperative outcomes

Mentions effect on range of motion

Discusses length of recovery

$J A M A$, GQS, and CFCS tools, ICC values were $>0.7$, indicating good interrater reliability.

\section{Statistical Methods}

All statistical tests were performed using Stata version 13.1 (StataCorp LC, College Station, TX). Descriptive statistics were used to quantify video characteristics, video reliability, and quality scores. Continuous variables are presented as means \pm standard deviations and ranges. Categorical variables are presented as relative frequencies with percentages. One-way analysis of variance (ANOVA) tests were used to determine if video reliability and quality differed based on video source and video content. Multivariate linear regression analyses were used to determine the influence of specific video characteristics on video reliability (JAMA score) and educational quality (GQS and CFCS). A $P$ value $<0.05$ was statistically significant.

\section{RESULTS}

Overall, 50 videos were analyzed, and the baseline characteristics of these videos are summarized in Table 4. The mean number of views per video was $124436.32 \pm 412883.32$. In total, the 50 included videos were viewed 6221816 times. The maximum number of views was 2591952 , and the minimum number of views was 163 .

The primary video content category was assessed for each video, and the results are summarized in Figure 1. The most common category represented was information about surgical technique (54\%). 
Table 4. Video characteristics.

\begin{tabular}{lccc}
\hline Characteristic & Mean \pm SD & Max & Min \\
\hline Video duration (min) & $10.9 \pm 18.27$ & 89.1 & 0.57 \\
Views & $124436.32 \pm 412883.32$ & 2591952 & 163 \\
Days since upload & $1733.57 \pm 1085$ & 4835 & 147 \\
View ratio (views/d) & $43.6 \pm 107.5$ & 536.1 & 0.17 \\
Likes & $232.5 \pm 860.1$ & 5600 & 0 \\
Dislikes & $22.7 \pm 92.4$ & 602 & 0 \\
Like ratio & $92 \pm 15.6$ & 100 & 0 \\
Video power index & $35.8 \pm 101.8$ & 470.5 & 0 \\
\hline
\end{tabular}

The lowest proportion of video content was attributed to exercise training, at $2 \%$. Video sources are summarized in Figure 2. Physicians were the most common source of video content (64\%), whereas patients were categorized as the least common source $(2 \%)$.

The mean JAMA, GQS, and CFCS scores, stratified by content and source, are summarized in Table 5. Overall, the mean JAMA score was 1.86 , the GQS was 1.48, and the CFCS was 5.28. In terms of the video sources, videos by physicians had the highest mean JAMA (2.1) and CFCS (5.8) scores. ANOVA demonstrated significant between-groups interactions in the $J A M A$ score $(P=0.042)$ between source categories, with physicians, academic sources, and medical sources having significantly higher mean JAMA scores. Analysis by content category revealed significant differences in the CFCS $(P=$ 0.018 ), with exercise training and nonsurgical management having higher mean scores. There were no other significant associations found between video source or content classification and the VPI, $J A M A$, or GQS.

Multivariate linear regression analyses were performed to determine if independent associations existed among video characteristics, video content category, video upload source, and video reliability and educational quality scores. Videos by physicians were significantly associated with higher JAMA scores $(\beta=0.616 ; P=0.025)$. In addition, videos

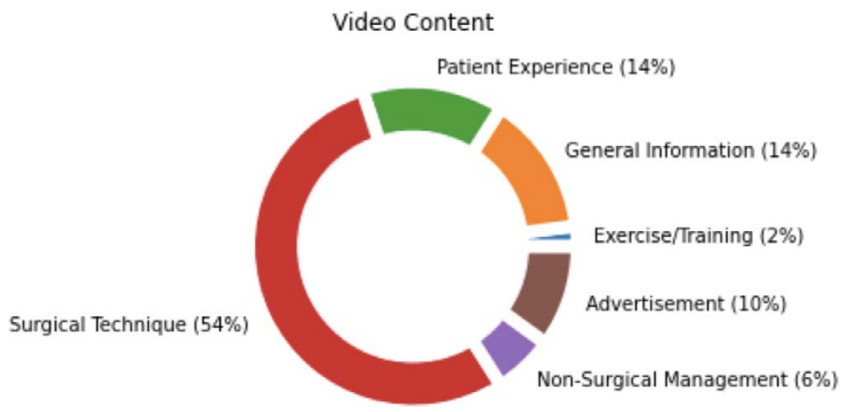

Figure 1. Videos stratification and percentage breakdowns by primary content material.

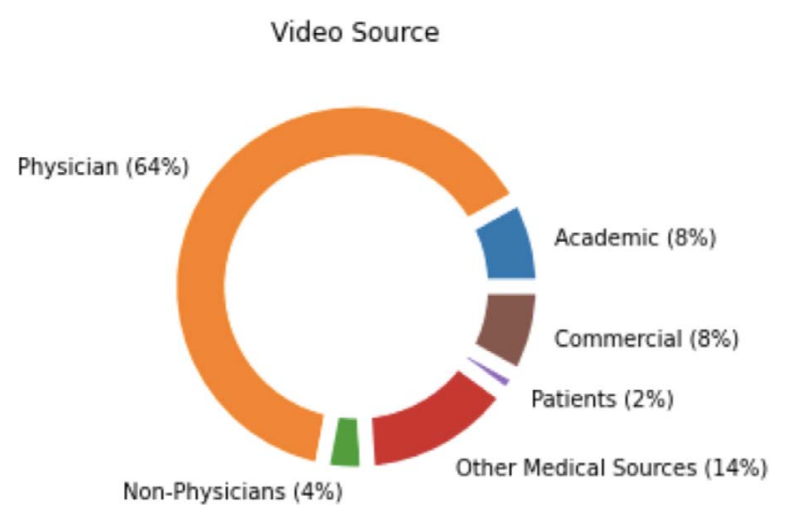

Figure 2. Video stratification and percentage breakdowns by publishing source.

classified as nonsurgical management were significant predictors of having higher JAMA $(\beta=0.907$; $P=0.050)$ and CFCS $(\beta=4.243 ; P=0.029)$ scores. Videos that were classified as advertisements were significant predictors of worse CFCS $(\beta=-3.978 ; P$ $=0.030)$, and videos by commercial sources were associated with significantly lower JAMA scores $(\beta$ $=-1.326 ; P=0.006)$.

\section{DISCUSSION}

This study sought to assess the quality and credibility of publicly available YouTube videos related to cervical fusion and video characteristics that were predictive of higher educational quality. Despite the popularity of the first 50 videos queried as denoted by total viewership, both the quality and educational content of the included videos was overall poor. Videos published by physicians were

Table 5. Quality and reliability of videos based on source and content.

\begin{tabular}{lccc}
\hline Variables & $\begin{array}{c}\boldsymbol{J} \boldsymbol{A M A} \boldsymbol{A}, \\
\text { Mean } \pm \text { SD }\end{array}$ & $\begin{array}{c}\text { GQS, } \\
\text { Mean } \pm \text { SD }\end{array}$ & $\begin{array}{c}\text { CFCS, } \\
\text { Mean } \pm \text { SD }\end{array}$ \\
\hline Overall $_{\text {Source }}^{\mathrm{a}}$ & $1.86 \pm 0.9$ & $1.48 \pm 0.7$ & $5.28 \pm 3.2$ \\
$\quad$ Academic & $1.8 \pm 1.0$ & $2.0 \pm 0.8$ & $4.3 \pm 3.3$ \\
$\quad$ Physician & $2.1 \pm 0.9$ & $1.4 \pm 0.7$ & $5.8 \pm 3.5$ \\
$\quad$ Nonphysicians & $1.0 \pm 0$ & $1.5 \pm 0.7$ & $4.5 \pm 3.5$ \\
$\quad$ Medical sources & $1.6 \pm 0.5$ & $1.6 \pm 0.8$ & $3.9 \pm 1.9$ \\
$\quad$ Patients & $1.0 \pm 0$ & $1.0 \pm 0$ & $5.0 \pm 0$ \\
$\quad$ Commercial & $1.0 \pm 0$ & $1.3 \pm 0.5$ & $5.3 \pm 3.0$ \\
Content & & & \\
$\quad$ Exercise or training & $3.0 \pm 0$ & $2.0 \pm 0$ & $10.0 \pm 0$ \\
$\quad$ General background & $1.6 \pm 0.8$ & $1.4 \pm 0.5$ & $5.9 \pm 3.1$ \\
$\quad$ Patient experience & $1.6 \pm 0.5$ & $1.1 \pm 0.4$ & $6.4 \pm 2.0$ \\
$\quad$ Surgical technique & $1.8 \pm 0.8$ & $1.5 \pm 0.7$ & $4.3 \pm 3.1$ \\
$\quad$ Nonsurgical management & $3.0 \pm 0$ & $1.7 \pm 0.6$ & $10.0 \pm 1.0$ \\
$\quad$ Advertisement & $2.4 \pm 1.3$ & $1.2 \pm 0.4$ & $4.0 \pm 2.9$
\end{tabular}

Abbreviations: JAMA, Journal of the American Medical Association; GQS, Global Quality Score; CFCS, Cervical Fusion Content Score.

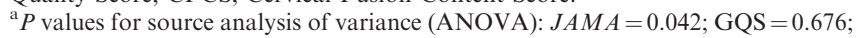
$\mathrm{CFCS}=0.765$.

${ }^{\mathrm{b}} P$ values for content ANOVA: $J A M A=0.055 ; \mathrm{GQS}=0.531 ; \mathrm{CFCS}=0.018$. 
independently associated with higher quality, while videos focusing on nonsurgical alternatives to cervical fusion were associated with higher quality and educational content. Commercial sources were associated with significantly lower quality, whereas videos intended as advertisements were associated with lower educational content, as assessed by the CFCS.

As anticipated, we observed that videos related to cervical fusion attracted many viewers. The total number of views for the videos included was 6221816 at the time of our analysis, with a mean of 124436.3 views. Previous orthopaedic and neurosurgical studies that aimed to assess the popularity of YouTube videos found comparable results. ${ }^{5,6,13}$ Kunze et $\mathrm{al}^{6}$ recently assessed the quality of videos related to posterior cruciate ligament injuries and reported a mean of 50477.9 views per video. Staunton et $\mathrm{al}^{5}$ in their 2015 study reported on videos related to scoliosis and found the mean number of views to be 71152 . More recently in 2018, Ovenden et $\mathrm{al}^{17}$ performed a similar query related to anterior cervical fusion and discectomy (ACDF), reporting a mean of 96239 views. The high number of views in our analysis further supports the notion that cervical fusion is a popular search topic for YouTube users and may attract a large patient viewership. Moreover, it may suggest that videos related to spinal disorders and procedures are becoming more popular over time; the mean number of views per video in our study is higher than the 2 previously mentioned studies.

While these videos may be popular, the overall quality and educational content were quite poor. This again is in line with previous studies. The aforementioned study of ACDF videos by Ovenden et $\mathrm{al}^{17}$ showed a mean $J A M A$ score of 1.63 . Brooks et $\mathrm{al}^{18}$ conducted a YouTube search for videos related to lumbar discectomy and found that only $19.8 \%$ of videos were rated as "good," while $49.4 \%$ were rated as "poor or inadequate." This trend of poor quality extends to a variety of other orthopaedic procedures and conditions outside the spine, including the knee and hip. ${ }^{3,4,6}$ Moreover, the mean CFCS, a tool we designed to assess the educational content quality for videos related to cervical fusion, was 5.28 out of a maximum of 16 , reflecting a substantial lack of pertinent information across all videos regardless of source.

Overall, we observed that a majority $(64 \%)$ of videos were produced by physicians. Previous studies have shown that videos produced by physicians are generally more reliable and of higher quality than videos produced by nonphysicians. ${ }^{19}$ In our analysis, we similarly found that videos produced by physicians, academic institutions, and other medical sources had significantly higher $J A M A$ scores relative to videos made by patients, nonphysicians, or commercial sources. Moreover, in our analysis, we found that videos produced by physicians were independently associated with higher quality videos. However, the educational content, as assessed by the GQS and CFCS, did not significantly differ between these groups. This suggests that, while the reliability of the videos produced by physicians and reputable medical sources may be higher, those videos are not necessarily of higher education quality or utility to patients.

On the other hand, videos produced by commercial sources were significantly associated with lower $J A M A$ scores. Furthermore, videos classified as advertisements were significantly associated with lower CFCS scores. Advertisements and direct-toconsumer marketing of medical therapies and procedures may lead patients to have skewed or unrealistic outcome expectations after procedures. ${ }^{20}$ In fact, Sherman et $\mathrm{al}^{21}$ analyzed pretreatment expectations in patients undergoing acupuncture for low back pain. They found that patients with higher pretreatment expectations generally had higher expectations for improvement, were less likely to pursue other treatment modalities, but were not more likely to have improved outcomes after treatment. In the context of cervical fusion, surgeons should attempt to assess patients' current understanding of surgery and should establish realistic patient expectations, particularly in those patients that have done independent research before a consultation.

Interestingly, analysis by content category did demonstrate that videos related to nonsurgical alternatives to surgery were independently associated with higher JAMA and CFCS scores. Overall, only $6 \%$ of videos analyzed were related to nonsurgical alternatives to surgery. Therefore, the statistical significance observed is likely a function of a low sample size. If the study sample was extended to include more videos, this relationship may not be evident. Alternatively, the question of whether to undergo surgery for spinal disorders is complex, and not everyone may benefit from surgery. ${ }^{22,23}$ There- 
fore, it is possible that the nonsurgical videos needed to include a more complete discussion of the relevant disease processes and surgical considerations to justify nonsurgical treatment.

This study has several limitations. First, only the top 50 videos returned were assessed. Therefore, many videos were excluded, and our analysis may not reflect the overall quality of videos available to patients. However, the top 50 videos are potentially the most important to evaluate, as these are the most likely to be found and viewed by patients. Second, the search terms cervical fusion used in our analysis may differ from terms used by patients. For example, patients may replace "cervical" with "neck" or other terms in their search, which may alter the returned videos. However, we believe our search terminology is broad enough to include the most popular videos directly pertinent to cervical surgery and reflect most videos that patients may encounter.

\section{CONCLUSIONS}

In this study, YouTube videos related to cervical fusion were assessed for reliability and educational quality. Overall, videos related to cervical fusion were popular and amassed a large viewership. However, they were poor in both quality and reliability. Videos by physicians were more associated with higher reliability scores relative to other sources. Videos produced by commercial sources and advertisements were associated with significantly lower reliability and educational content scores. Currently, YouTube seems to be an unreliable source of information on cervical fusion for patients. Surgeons should carefully review pertinent information related to cervical fusion with patients to clarify and correct any misinformation to establish realistic surgical expectations.

\section{REFERENCES}

1. Frost JH, Massagli MP. Social uses of personal health information within PatientsLikeMe, an online patient community: what can happen when patients have access to one another's data. J Med Internet Res. 2008;10(3):e15. doi:10.2196/ jmir. 1053

2. Shaw RJ, Johnson CM. Health information seeking and social media use on the Internet among people with diabetes. Online J Public Health Inform. 2011;3(1). doi:10.5210/ojphi. v3i1.3561

3. MacLeod MG, Hoppe DJ, Simunovic N, Bhandari M, Philippon MJ, Ayeni OR. YouTube as an information source for femoroacetabular impingement: a systematic review of video content. Arthroscopy. 2015;31(1):136-142.

4. Wong M, Desai B, Bautista M, Kwon O, Kolodychuk N, Chimento G. YouTube is a poor source of patient information for knee arthroplasty and knee osteoarthritis. Arthroplast Today. 2019;5(1):78-82.

5. Staunton PF, Baker JF, Green J, Devitt A. Online curves: a quality analysis of scoliosis videos on YouTube. Spine. 2015;40(23):1857-1861.

6. Kunze KN, Cohn MR, Wakefield C, et al. YouTube as a source of information about the posterior cruciate ligament: a content-quality and reliability analysis. Sports Med Arthrosc Rehabil Ther Technol. 2019;1(2):e109-e114.

7. Koenig S, Nadarajah V, Smuda MP, Meredith S, Packer JD, Frank Henn R. Patients' use and perception of Internetbased orthopaedic sports medicine resources. Orthop J Sports Med. $2018 ; 6(9): 232596711879646$. doi:10.1177/ 2325967118796469

8. Lehto IJ, Tertti MO, Komu ME, Paajanen HEK, Tuominen J, Kormano MJ. Age-related MRI changes at 0.1 $\mathrm{T}$ in cervical discs in asymptomatic subjects. Neuroradiology. 1994;36(1):49-53. doi:10.1007/bf00599196

9. Matsumoto M, Fujimura Y, Suzuki N, et al. MRI of cervical intervertebral discs in asymptomatic subjects. $J$ Bone Joint Surg Br. 1998;80(1):19-24.

10. Boden SD, McCowin PR, Davis DO, Dina TS, Mark AS, Wiesel S. Abnormal magnetic-resonance scans of the cervical spine in asymptomatic subjects. A prospective investigation. J Bone Joint Surg Am. 1990;72(8):1178-1184.

11. Radhakrishnan K, Litchy WJ, O'Fallon WM, Kurland LT. Epidemiology of cervical radiculopathy. A populationbased study from Rochester, Minnesota, 1976 through 1990. Brain. 1994;117(Pt 2):325-335.

12. Oglesby M, Fineberg SJ, Patel AA, Pelton MA, Singh K. Epidemiological trends in cervical spine surgery for degenerative diseases between 2002 and 2009. Spine. 2013;38(14):12261232.

13. Erdem MN, Karaca S. Evaluating the accuracy and quality of the information in kyphosis videos shared on YouTube. Spine. 2018;43(22):E1334-E1339.

14. Cassidy JT, Fitzgerald E, Cassidy ES, et al. YouTube provides poor information regarding anterior cruciate ligament injury and reconstruction. Knee Surg Sports Traumatol Arthrosc. 2018;26(3):840-845.

15. Park DK. Spinal Fusion. OrthoInfo. Published June 2018. https://orthoinfo.aaos.org/en/treatment/spinal-fusion/ Accessed September 30, 2020.

16. Wang D, Jayakar RG, Leong NL, Leathers MP, Williams RJ, Jones KJ. Evaluation of the quality, accuracy, and readability of online patient resources for the management of articular cartilage defects. Cartilage. 2017;8(2):112-118.

17. Ovenden CD, Brooks FM. Anterior cervical discectomy and fusion YouTube videos as a source of patient education. Asian Spine J. 2018;12(6):987-991.

18. Brooks FM, Lawrence H, Jones A, McCarthy MJH. YouTube $^{\mathrm{TM}}$ as a source of patient information for lumbar discectomy. Ann R Coll Surg Engl. 2014;96(2):144-146.

19. Tartaglione JP, Rosenbaum AJ, Abousayed M, Hushmendy SF, DiPreta JA. Evaluating the quality, accuracy, and readability of online resources pertaining to hallux valgus. Foot Ankle Spec. 2016;9(1):17-23.

20. Paraskeva N. Media, marketing and mass advertising: 
managing patient expectations. J Aesthet Nurs. 2015;4(5):236237.

21. Sherman KJ, Cherkin DC, Ichikawa L, et al. Treatment expectations and preferences as predictors of outcome of acupuncture for chronic back pain. Spine. 2010;35(15):1471.

22. Cheung JPY, Luk KD-K. Complications of anterior and posterior cervical spine surgery. Asian Spine J. 2016;10(2):385400.

23. Zaina F, Tomkins-Lane C, Carragee E, Negrini S. Surgical versus non-surgical treatment for lumbar spinal stenosis. Cochrane Database Syst Rev. 2016;(1):CD010264. doi:10.1002/14651858.CD010264.pub2.

Disclosures and COI: Each author certified that he or she has no commercial associations (eg, consultancies, stock ownership, equity interest, patent or licensing arrangements) that might pose a conflict of interest in connection with the submitted article. This study was approved by the
Institutional Review Board at Rush University Medical Center.

Corresponding Author: Arash J. Sayari, MD, Department of Orthopaedic Surgery, Division of Spine Surgery, Rush University Medical Center, 1611 West Harrison Street, Chicago, IL 60612. Phone: (312) 942-5000; Email: arashsayari@gmail. com.

Published 19 August 2021

This manuscript is generously published free of charge by ISASS, the International Society for the Advancement of Spine Surgery. Copyright (C) 2021 ISASS. To see more or order reprints or permissions, see http://ijssurgery.com. 